\title{
Probing Majorana Neutrinos at the CMS
}

\section{SungBin Oh*}

on behalf of the CMS Collaboration

Seoul National University

E-mail: sungbin.oh@cern.ch

\begin{abstract}
We report a search for heavy Majorana neutrinos $(\mathrm{N})$ decaying into a $\mathrm{W}$ boson and a lepton using the CMS detector at the Large Hadron Collider. We search for a signature of two samesign electrons and two jets or two same-sign muons and two jets or a same-sign electron-muon pair and two jets using $19.7 \mathrm{fb}^{-1}$ of data collected during 2012 in proton-proton collisions at a center-of-mass energy of $8 \mathrm{TeV}$. We find the data to be consistent with the expected standard model background and upper limits are set on $\left|V_{I N}\right|^{2}$, as a function of heavy Majorana neutrino mass from $40-500 \mathrm{GeV} / c^{2}$, where $V_{l N}$ is the mixing element of the heavy Majorana neutrino with the standard model neutrino. The upper limits obtained for $\left(\left|V_{e N}\right|^{2},\left|V_{\mu N}\right|^{2},\left|V_{e N} V_{\mu N}^{*}\right|\right)$ are $(0.022$, $0.00470,0.009)$ for $m_{N}=50 \mathrm{GeV} / c^{2},(0.016,0.0123,0.009)$ for $m_{N}=200 \mathrm{GeV} / c^{2}$, and $(0.72$, $0.583,0.47$ ) for $m_{N}=500 \mathrm{GeV} / c^{2}$. These results extend considerably the regions excluded by previous direct searches.
\end{abstract}

38th International Conference on High Energy Physics 3-10 August 2016

Chicago, USA

${ }^{*}$ Speaker. 


\section{Introduction}

The discovery of neutrino oscillation established that neutrinos have non-zero masses. Results from various neutrino oscillation experiments and cosmological constraints showed the upper limit of neutrino mass sum is very small, less than $0.13 \mathrm{eV}$.

The so-called "SeeSaw" mechanism is able to explain this smallness of neutrino masses in natural way by introducing a heavy neutrino state $\mathrm{N}$. Here, the mass of standard neutrino is given by $m_{v} \sim y_{v}^{2} v^{2} / m_{N}$, where $y_{v}$ is a Yukawa coupling, $v$ is the Higgs vacuum expectation value in the standard model (SM) and $m_{N}$ is the mass of the heavy Majorana neutrino state. Direct searches of heavy Majorana neutrinos are very important to understand the nature of neutrinos and the origin of their masses. We considered a heavy Majorana neutrino mixes with the SM neutrinos, with $m_{N}$ and $V_{l N}$ as free parameters of the model. Here $V_{l N}$ is a mixing element describing the mixing between the heavy Majorana neutrino and the SM neutrino of flavour $l$.

In the process we performed the search, the heavy Majorana neutrino is produced by s-channel production of a $\mathrm{W}$ boson, which dacays via $W^{ \pm} \rightarrow N l^{ \pm}$. The $\mathrm{N}$ is assumed to be a Majorana particle, so it can decay via $N \rightarrow W^{\mp} l^{ \pm}$with $W^{\mp} \rightarrow q \bar{q}^{\prime}$, resulting in a $l^{ \pm} l^{ \pm}+q \bar{q}^{\prime}$ final state.

\section{CMS detector and Event Selection}

The central feature of the CMS detector is a superconducting solenoid with an internal radius of $3 \mathrm{~m}$. The solenoid provides a magnetic field of $3.8 \mathrm{~T}$ along the direction of the counter-clockwise rotating beam. Within the superconducting solenoid volume are a silicon pixel and strip tracker, a lead tungstate crystal electromagnetic calorimeter (ECAL), and a brass and scintillator hadron calorimeter (HCAL), each composed of a barrel and two endcap sections. Muons are measured in gas ionization detectors embedded in the steel return yoke. Extensive forward calorimetry complements the coverage provided by the barrel and endcap detectors. The first level of the CMS trigger system, composed of custom hardware processors, uses information from the calorimeters and muon detectors to select up to $100 \mathrm{kHz}$ of the most interesting events. The High Level Trigger (HLT) processor farm uses information from all CMS subdetectors to further decrease the event rate to roughly $300 \mathrm{~Hz}$ before data storage. A more detailed description of the CMS detector can be found in [3].

In the analysis, we use several samples of simulated SM process which include a full treatment of the pp collisions. Theses samples include the full simulation of the CMS detector based on GEANT4 and are reconstructed using the same CMS software as used for data. In order to simulate heavy Majorana neutrino events, we use a leading-order (LO) event generator describes in [4], which was implemented in ALPGEN v2.14 [5] with the CTEQ6M parton distribution functions (PDFs) [7]. We assumed $\left|V_{l N}\right|^{2}=1$, and considered mass of heavy Majorana neutrino from 40 to $500 \mathrm{GeV} / c^{2}$ [11]. We scale the $\mathrm{LO}$ cross section by a factor of 1.34 to account for higher-order corrections, based on the next-to-next-to-leading-order calcuation in FEWZ [9, 10]. Parton showering 
and hadronization are simulated using PYTHIA v6.4.22 [8].

Additional selections are applied to the triggered events to ensure the presence of well-identified dileptons and jets. In this analysis, we concentrate on the same-sign (SS) dilepton and additional two jets without missing any final state energy from the heavy Majorana neutrino decay. Lepton candidates are required to pass CMS official tight ID. Additionally, we used more tight relative isolation cut to reduce fake lepton background from QCD processes. The invariant mass of the dilepton pair is required to be above $10 \mathrm{GeV} / \mathrm{c}^{2}$ and outside the $\mathrm{Z}$ mass peak for the dielectron channel, to remove a significant amount of background from charge mismearsurement. We also veto third lepton to suppress background from diboson production events. Jets are selected with CMS official selection. To reduce background from top quark decays, b-quark jet veto was applied where the medium working point of the combined secondary vertex tagger [12] has been used.

Depending on the heavy Majorana neutrino mass, signal events from heavy Majorana neutrino decays have different kinematic properties. In the low neutrino mass search region $\left(m_{N}<m_{W}\right)$, the W-boson propagator is on-shell and the final state system of $M\left(l^{ \pm} l^{ \pm} j j\right)$ should be close to the $\mathrm{W}$ mass. In the high neutrino mass search region $\left(m_{N}>m_{W}\right)$, the W-boson propagator is offshell but the W-boson from the heavy Majorana neutrino decay is on-shell, so the $M(j j)$ will be close to the $\mathrm{W}$ mass. We require missing transverse energy (MET) to be less than 30 (35) GeV in low-mass (high-mass) search region because we have no neutrino at final state of signal process. We applied additional cuts to reduce background without losing many portion of signal events. Detailed information about event selection can be found in $[1,2]$.

\section{Background Estimation and Systematic Uncertainties}

There are three major sources of same-sign dilepton backgrounds. The first and the most important background events are originated from misidentification (fake) of objects as prompt leptons. These "prompt leptons" originate from B hadron decays, light-quark or gluon jets as well as from photon conversions, and are typically not well isolated. The simulation is not reliable because of lack of statistics comes from small misidentification probability. Data driven method from QCD enriched data sample was used to estimate fake lepton background [1,2].

The second contribution is from SM production of two genuine isolated leptons of the same charge. This irreducible background consist of diboson events as well as triboson and $t \bar{t}$ plus boson, other rare processes include Higgs event where Higgs decays into neutral bosons, or associated Higgs production $\mathrm{VH}$ and $t \bar{t} \mathrm{H}$ in which case also $\mathrm{H} \rightarrow \mathrm{WW}$ contribute. We estimate the background using PYTHIA genertator and MADGRAPH v5.1.3.30 [6].

The third background source is from opposite-sign event in which the charge of one of the electrons is mismeasured. We used both simulated sample of Drell-Yan process and data to evaluate the charge mismeasurement probability as a function of Pt and $\eta$ of the electron. The charge mismeasurement background of muon is ignored because of much low probability $[1,2]$. 
The main source of systematic uncertainties are associated with the background estimates. The overall systematic uncertainty on the misidentified lepton background is $40 \%, 28 \%$ and $35 \%$ for dielectron, dimuon and electron-muon channels respectively. An uncertainty of $12 \%$ assigned to the background from charge mis-measurement for dielectron channel. The remaining candidates are cross section uncertainty of MC samples and detector effect. Detailed information about systematic uncertainty can be found in $[1,2]$.

\section{Results and Summary}

We see no evidence for a significant excess in the data beyond the backgrounds predicted from SM and we set 95\% confidence level (CL) exclusion limits on the square of the Majorana neutrino mixing element and cross section times branching fraction for $\mathrm{pp} \rightarrow N l^{ \pm} \rightarrow l^{ \pm} l^{ \pm} q \bar{q}^{\prime}$ as a function of $m_{N}$. We use Poisson distributions for the signal and log-normal distributions for the nuisance parameters. For simple interpretation, each channel of dielectron, dimuon, electron-muon channels is considered as independent analysis to each other. We set limits on $\left|V_{e N}\right|^{2},\left|V_{\mu N}\right|^{2}$, and $\left|V_{e N} V_{\mu N}^{*}\right|$ as a function of $m_{N}$.

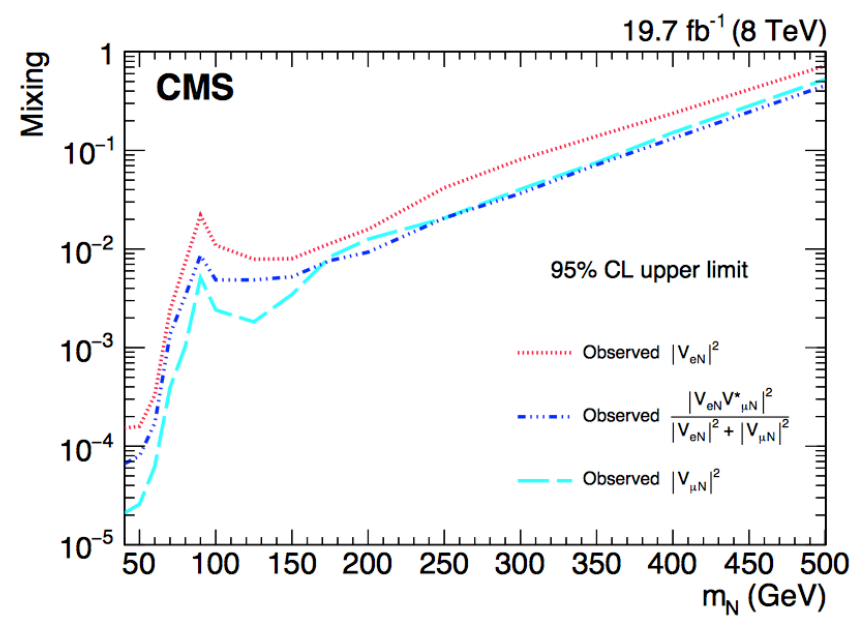

Figure 1: 95\% CL limits on mixing parameter between the heavy Majorana neutrino and SM neutrinos

We have performed a search for heavy Majorana neutrinos decaying into same-sign leptons and two jets using $19.7 \mathrm{fb}^{-1}$ of data collected during 2012 in pp collisions at a center-of-mass energy of $8 \mathrm{TeV}$. We do not observe any excess of events beyond the expected standard model background and set 95\% CL upper limits on the mixing elements, $\left|V_{e N}\right|^{2},\left|V_{\mu N}\right|^{2}$, and $\left|V_{e N} V_{\mu N}^{*}\right|$, of the Majorana neutrino as a function of Majorana neutrino mass from $40 \mathrm{GeV} / c^{2}$ to $500 \mathrm{GeV} / c^{2}$.

The results significantly improve the previous LHC limits on $\left|V_{l N}\right|^{2}$ as well as the studied mass hypothesis range. 


\section{Acknowledgement}

This research was supported by the National Research Foundation of Korea ( NRF-2015R1A4A1042542 and NRF-2013R1A2A2A01069162)

\section{References}

[1] CMS Collaboration, "Search for heavy Majorana neutrinos in $\mu^{ \pm} \mu^{ \pm}+$jets events in proton-proton collisions at $\sqrt{s}=8 \mathrm{TeV}$ ", Phys. Lett. B 748 (2015) 144

[2] CMS Collaboration, "Search for heavy Majorana neutrinos in $e^{ \pm} e^{ \pm}+$jets and $e^{ \pm} \mu^{ \pm}+$jets events in proton-proton collisions at $\sqrt{s}=8 \mathrm{TeV}$ ", JHEP 04 (2016) 169

[3] CMS Collaboration, "The CMS experiment at the CERN LHC", JINST 3 (2008) S08004, doi:10.1088/1748-0221/3/08/S08004.

[4] F. del Aguila, J. Aguilar-Saavedra, and R. Pittau, "Heavy neutrino signals at large hadron colliders", JHEP 0710 (2007) 047

[5] M. L. Mangano et al., "ALPGEN, a generator for hard multiparton processes in hadronic collisions", JHEP 0307 (2003) 001,

[6] J. Alwall et al., "MadGraph 5 : Going Beyond”, JHEP 1106 (2011) 128

[7] J. Pumplin et al., "New generation of parton distributions with uncertainties from global QCD analysis", JHEP 0207 (2002) 012

[8] T. Sjostrand, S. Mrenna, and P. Z. Skands, ”PYTHIA 6.4 Physics and Manual”, JHEP 0605 (2006) 026

[9] R. Gavin, Y. Li, F. Petriello, and S. Quackenbush, "FEWZ 2.0: A code for hadronic Z production at next-to-next-to-leading order", Comput. Phys. Commun. 182 (2011) 2388-2403

[10] R. Gavin, Y. Li, F. Petriello, and S. Quackenbush, ”W Physics at the LHC with FEWZ 2.1”, Comput. Phys. Commun. 184 (2013) 208-214

[11] F. del Aguila, J. Aguilar-Saavedra, and R. Pittau, "Heavy neutrino signals at large hadron colliders", JHEP 0710 (2007) 047,

[12] CMS Collaboration, "Identification of b-quark jets with the CMS experiment", JINST 8 (2013) $\mathrm{P} 04013$ 